\title{
Atenção do cirurgião-dentista da estratégia de saúde da família no atendimento domiciliar à pacientes acamados: revisão de literatura
}

Attention of the family health strategy dentist in home care for bedridden patients: a literature review Atención de lo dentista de la estrategia de salud familiar en los cuidados en el hogar

para los pacientes prostrados en cama: revisión de literatura

Sara de Oliveira ROSA ${ }^{1}$

Iago RAMIREZ1

Daniela Coelho DE LIMA2

Alessandro Aparecido PEREIRA2

${ }^{1}$ Residente em Saúde da Família, Programa de Residência Multiprofissional em Saúde da Família, Escola de Enfermagem, Universidade Federal de Alfenas (UNIFAL-MG), 37130-001 Alfenas-MG, Brasil

${ }^{2}$ Docente do Departamento de Clínica e Cirurgia da Faculdade de Odontologia, Universidade Federal de Alfenas (UNIFAL-MG), 37130-001 Alfenas-MG, Brasil

\section{Resumo}

A Estratégia de Saúde da Família (ESF) faz parte do Sistema Único de Saúde (SUS), e dentro dela uma das práticas preconizadas é a atenção domiciliar (AD) com objetivo principal fornecer continuidade do cuidado. Na equipe multiprofissional uma das atribuições para o cirurgião-dentista (CD) previsto na Política Nacional de Atenção Básica (PNAB) é o cuidado no domicílio. O objetivo desse estudo é conhecer as atribuições dos cirurgiões-dentistas da ESF referente à importância das visitas domiciliares aos pacientes acamados, dando ênfase em quais os procedimentos os CD's podem realizar no domicílio sem o equipamento portátil e analisar pesquisas de campo sobre visitas domiciliares realizadas por cirurgiões-dentistas. Porém, quanto à relevância profissional, a literatura evidencia lacunas a essas visitas realizadas pelos CD's. O trabalho desenvolvido trata-se de uma revisão integrativa, em que os artigos científicos sobre a temática foram acessados nas bases de dados Scielo, LILACS, MEDLINE, publicados de 2004 até 2020. O AD é um recurso útil para a Saúde Bucal se bem delimitada, como em situações cujo a dinâmica familiar interfere no processo saúde-doença. Além de estabelecer ou fortalecer o vínculo, pode ser utilizada para intervenções curativas nos pacientes impossibilitados de se deslocarem até a Unidade Básica de Saúde (UBS). E quando a visita domiciliar não é vivenciada frequentemente no cotidiano do trabalho na Atenção Básica, o vínculo com a população adscrita fica prejudicado, o que retroalimenta o ciclo do processo de trabalho alienante e isolado dos profissionais da saúde bucal.

Descritores: Repouso em Cama; Saúde da Família; Pacientes Domiciliares; Consulta a Domicílio.

\section{Abstract}

The Family Health Strategy (ESF) is part of the Unified Health System (SUS), and within it one of the recommended practices is home care, whose main objective is to provide continuity of care. Inside the multidisciplinary team, one of the duties for the dental surgeon (CD) foreseen in the National Primary Care Policy (PNAB) is the home care. The objective of this study is to know the attributions of FHS dentists regarding the importance of home visits to bedridden patients, emphasizing which procedures CD's can perform at home without portable equipment and to analyze field research on home visits carried out by dental surgeons. However, as for professional relevance, the literature shows gaps in these visits made by the CD's. The carried-out study is an integrative review, in which the scientific articles on the theme were accessed in the online databases Scielo, LILACS, MEDLINE, published from 2004 to 2020. AD was shown to be an useful resource for Oral Health, if well defined, as in situations where family dynamics interfere with the health-disease process. In addition to establishing or strengthening the bond, it can be used for curative interventions in patients unable to travel to the Basic Health Unit (UBS). And when home visits are not frequently experienced in the daily work of Primary Care, the bond with the registered population is impaired, which feeds back into the cycle of the alienating and isolated work process of oral health professionals.

Descriptors: Bed Rest; Family Health; Homebound Persons; House Calls.

\section{Resumen}

La Estrategia de Salud de Familiar (ESF) forma parte del Sistema Único de Salud (SUS), y dentro de él, una de las prácticas recomendadas es la atención domiciliaria (AD) con el objetivo principal de brindar continuidad en la atención. En el equipo multiprofesional, una de las funciones del cirujano dentista (CD) previstas en la Política Nacional de Atención Primaria (PNAB) es la atención domiciliaria. El objetivo de este estudio es conocer las atribuciones de los odontólogos de la ESF sobre la importancia de las visitas domiciliarias a los pacientes encamados, enfatizando qué procedimientos pueden realizar los CD's en casa sin equipo portátil y analizar la investigación de campo sobre visitas domiciliarias realizadas por cirujanos dentistas. Sin embargo, en cuanto a relevancia profesional, la literatura muestra lagunas en estas visitas realizadas por los CD's. El trabajo desarrollado es una revisión integradora, en la que se accedió a los artículos científicos sobre el tema en las bases de datos Scielo, LILACS, MEDLINE, publicados desde 2004 a 2020. La EA es un recurso útil para la Salud Bucal, si bien definida, como en situaciones donde la dinámica familiar interfiere con el proceso salud-enfermedad. Además de establecer o fortalecer el vínculo, se puede utilizar para intervenciones curativas en pacientes que no pueden desplazarse a la Unidad Básica de Salud (UBS). Y cuando las visitas domiciliarias no se viven con frecuencia en el quehacer diario de Atención Primaria, se deteriora el vínculo con la población censada, lo que alimenta el ciclo del proceso de trabajo alienante y aislado de los profesionales de la salud bucodental.

Descriptores: Reposo en Cama; Salud de la Familia; Personas Imposibilitadas; Consulta Médica a Domicilio.

INTRODUÇÃO

$$
\text { O Sistema Único de Saúde (SUS) é o }
$$
modelo de saúde vigente no Brasil que se organiza de forma regionalizada e hierarquizada, e se caracteriza pela combinação de serviços e ações de promoção, prevenção e recuperação da saúde. Tal modelo estabelece a Atenção Primária à Saúde (APS) como a principal ordenadora da rede de atenção. A organização da prática da APS é realizada por meio da Estratégia Saúde da Família (ESF), sendo executada pela implantação de Unidades Básicas de Saúde (UBS), baseadas em uma equipe multiprofissional responsável pelo cuidado e acompanhamento de um número de famílias localizadas em sua área de cobertura".

Tal equipe é composta no mínimo por 
médico, enfermeiro, técnico e/ou auxiliar de enfermagem e agente comunitário de saúde (ACS). Ainda podem complementar a equipe o agente de combate às endemias (ACE), cirurgião-dentista e técnico e/ou auxiliar em saúde bucal. Uma das atribuições comuns a todos os integrantes da equipe de saúde é a oferta do cuidado integral à saúde da população inserida no território da Unidade Básica de Saúde, e no domicílio e em espaços comunitários (creches, associações, escolas, etc.), com um enfoque especial nas condições das populações que possuem necessidades específicas (moradores de rua, jovens em medida socioeducativa, privados do direito de ir e vir, população ribeirinha e fluvial, etc.) ${ }^{2}$.

Tendo em foco o cuidado no domicílio, a Atenção Domiciliar (AD) compõe uma modalidade de atenção à saúde que pode substituir ou complementar as modalidades existentes, sendo caracterizada por uma combinação de ações de promoção da saúde, prevenção dos agravos, tratamento e reabilitação de doenças que são executadas no domicílio $^{3}$. Tendo em foco o cuidado no domicílio, a Atenção Domiciliar (AD) compõe uma modalidade de atenção à saúde que pode substituir ou complementar as modalidades existentes, sendo caracterizada por uma combinação de ações de promoção da saúde, prevenção dos agravos, tratamento e reabilitação de doenças que são executadas no domicílio ${ }^{4}$. Por isso a saúde bucal do paciente acamado deve ser avaliada constantemente, pois sua condição oral interfere diretamente em sua qualidade de vida ${ }^{5}$. E a falta do acompanhamento do profissional odontólogo pode acarretar em quadros precários de saúde oral que incluem cáries extensas levando ao surgimento de raízes residuais, doenças periodontais e desordens potencialmente malignas ${ }^{4}$.

Porém, dificilmente o CD consegue sair do consultório da ESF para ir até um domicílio prestar seu serviço, isso ocorre devido a alta demanda de atendimentos clínicos e principalmente demandas espontâneas nas unidades de saúde ${ }^{6}$, além deles não terem uma agenda organizada para ambos os problemas, no que se diz respeito das visitas e dos atendimentos nos consultórios ${ }^{5}$. Com isso, percebe-se que quase metade dos profissionais de saúde bucal não realizam o cuidado no domicílio, contabilizando uma quantidade de visitas bem menor em relação aos demais profissionais de saúde da Unidade de Saúde, principalmente enfermeiros, médicos e técnicos/auxiliares de enfermagem ${ }^{5}$.
O cuidado em Saúde Bucal, na esfera da Atenção Domiciliar possibilita a propagação de ações em promoção da saúde e prevenção de patologias relacionadas à cavidade bucal, e ainda permite a realização de procedimentos odontológicos no próprio domicílio, promovendo um cuidado que eleve a autonomia e estreite o relacionamento entre o cuidador e seu paciente, sendo dessa forma indispensável para a manutenção da qualidade de vida do mesmo ${ }^{7}$. Desse modo, essa revisão buscou conhecer as atribuições dos cirurgiões-dentistas da ESF preconizadas pela Política Nacional de Atenção Básica (PNAB) sobre a importância das visitas domiciliares aos pacientes acamados, e com isso analisar quais pacientes se enquadram como acamados, quais as principais alterações bucais nestes pacientes e perceber a presença de pesquisas de campo sobre visitas domiciliares realizadas por dentistas.

MATERIAL E MÉTODO

O trabalho desenvolvido seguiu os preceitos do estudo exploratório, dissertativo, por meio de uma pesquisa bibliográfica, conforme Gil et al. ${ }^{8}$. Os artigos científicos sobre a temática foram acessados nas bases de dados Scielo, LILACS, MEDLINE, publicados nos últimos 13 anos, de 2007 até 2020.

Ao total foram 23 artigos científicos selecionados para realização dessa pesquisa. Para a seleção dos mesmos, foram consideradas como critério de inclusão as bibliografias que abordassem as visitas domiciliares realizadas por cirurgiões-dentistas nas Estratégia de Saúde da Família (ESF), e foram excluídas aquelas que não atenderam a temática, como por exemplo os profissionais de redes privadas.

A coleta de dados seguiu a seguinte premissa: análise dos trabalhos por título e resumo, objetivando verificar se ia de acordo com os critérios de elegibilidade deste trabalho, seleção dos artigos por leitura de texto completo dos artigos acessados na íntegra; registro das informações extraídas das fontes em instrumento específico (autores, ano, método, resultados e conclusões). Para análise e interpretação, foi realizada uma leitura crítica com a finalidade de ordenar e sintetizar as informações contidas nas fontes, de forma que estas possibilitassem a obtenção de respostas à questão-problema da pesquisa.

RESULTADOS

Frente à extração de dados realizada nos artigos incluídos na revisão, pode-se destacar alguns dados relevantes à temática da revisão, sintetizadas principalmente na relação 
da Equipe de Saúde Bucal da ESF com a AD, os principais desafios relatados e as de que formas as VDs ocorrem, e/ou por quais razões deixam de ocorrer.

Uma das práticas preconizadas pela Estratégia de Saúde da Família (ESF) é a atenção domiciliar (AD), e esta pode ser identificada como um conjunto de ações e prestações de serviços relacionados à promoção, prevenção e tratamento de doenças, como também reabilitação prestadas em domicílio, com objetivo de fornecer a continuidade do cuidado, além de ser integrada às redes de atenção à saúde ${ }^{2}$. Apesar da Atenção Primária à Saúde estar bem estabelecida nas últimas duas décadas, tem-se notado que o cuidado domiciliar encontra-se negligenciado e sobrecarregado devido à alta demanda por cuidados que excedem a capacidade de atendimento da equipe ${ }^{10}$.

As visitas domiciliares tem como objetivos realizar a avaliação de risco, o diagnóstico, a educação em saúde, o encaminhamento para tratamento e, quando necessário, a assistência odontológica aos usuários com limitação funcional da área de abrangência ${ }^{11}$. Elas são essenciais para se criar fortes vínculos entre pessoa-familiar-equipe multiprofissional, principalmente entre pessoas com dificuldades locomotoras ${ }^{12}$. Para tanto, a PNAB determina como uma das atribuições das equipes de $A D$ a assistência às pessoas e seus familiares que apresentem dificuldades de acesso aos serviços de saúde ${ }^{13}$.

A Atenção Domiciliar se divide em três modalidades, sendo estas respectivamente: Atenção Domiciliar 1 (AD1), Atenção Domiciliar 2 (AD2) e Atenção Domiciliar 3 (AD3). A Atenção Domiciliar 1 exerce sua prestação de assistência à saúde por meio do acompanhamento linear no domicílio e está sob a supervisão e execução da Atenção Básica (AB). A AB deverá ser amparada pelos Núcleos de Apoio à Saúde da Família (NASF), ambulatórios de especialidades e centros de reabilitação na oferta do cuidado. Já a Atenção Domiciliar 2 (AD2) e a Atenção Domiciliar 3 (AD3) serão de responsabilidade do Serviço de Atenção Domiciliar (SAD), que é um serviço substitutivo ou complementar à internação hospitalar ou ao atendimento ambulatorial ${ }^{14}$.

Quanto aos pacientes acamados, esses são na maioria das vezes idosos portadores de diversas patologias como hipertensão arterial, cardiopatias, diabetes, doença de Alzheimer, pneumonia, endocardite bacteriana e outras doenças relacionadas à idade avançada, e por isso necessitam de um acompanhamento longitudinal por parte da equipe que está inserida na ESF ${ }^{3}$. Muitos desses usuários se encontram em situação econômica desfavorável e em vulnerabilidade social, além de necessitarem de auxílio em suas atividades básicas e instrumentais do dia-a-dia ${ }^{15}$.

Outros usuários que também dependem de cuidados no domicílio são aqueles que tem limitação funcional e/ou doenças graves, obesos mórbidos, restritos ao leito e/ou domicílio, em que o deslocamento até a unidade de saúde torna-se impeditivo de acesso ao atendimento odontológico, bem como usuários e/ou famílias em condições de vulneração ${ }^{11}$. Para tanto, é importante ressaltar que $O A D$ é responsável pelo atendimento relacionado à visita e a internação domiciliar, sendo esta realizada por uma equipe multiprofissional de saúde, com o objetivo de possibilitar uma assistência em saúde humanizada, holística e permeada de vínculo. Neste contexto, a visita domiciliar pode ser entendida como porta de acesso da pessoa às ações e serviços de saúde da ESF ${ }^{18}$.

O cuidado no domicílio está entre as atribuições previstas para o profissional $C D$ na PNAB, onde fica prevista a realização da atenção em saúde bucal seja na unidade, espaços comunitários ou domicílio, englobando todas as esferas (individual e coletiva) e nas diversas linhas de ação (promoção, prevenção, proteção da saúde e tratamento, acompanhamento, manutenção e reabilitação da saúde) ${ }^{2}$. A Política Nacional de Saúde Bucal intensifica a importância da equipe de saúde bucal realizar as visitas domiciliares às pessoas com dificuldade de deslocamento à ESF, de forma a ampliar seu acesso ${ }^{19}$. Com o avançar da idade há uma certa prevalência de DCNT na população, e consequentemente um uso elevado de diversos medicamentos para o tratamento dessas patologias. O somatório da presença de DCNT, associadas ao alto uso de medicações para seu tratamento, aliada a dificuldade em realizar a higiene bucal de forma correta e satisfatória e a exposição a diversos fatores etiológicos de patologias bucais, culmina na instalação de diversas doenças do complexo bucal ${ }^{17}$.

As principais alterações bucais encontradas nos pacientes que necessitam de cuidados domiciliares incluem: xerostomia, disgeusia, hipossalivação, estomatites devido ao uso de próteses desadaptadas, candidíase eritematosa, queilite angular, cárie, doenças periodontais, edentulismo, língua geográfica e saburrosa. Portanto, cabe ao CD à orientação e supervisão de pacientes e familiares em relação aos cuidados com a higiene bucal através da 
criação de uma rotina de higiene oral, e a realização de procedimentos pouco traumáticos ${ }^{17}$.

O atendimento domiciliar é um recurso útil para a Saúde Bucal se bem delimitada, como em situações cujo a dinâmica familiar interfere no processo saúde-doença e a presença do $C D$ in loco traz benefícios na compreensão da realidade do usuário. Além de estabelecer ou fortalecer o vínculo como citados anteriormente, também pode ser utilizada para intervenções curativas nos pacientes impossibilitados de se deslocarem até a USB, isso quando há disponibilidade de equipamentos para atendimentos home-care ${ }^{1}$.

Por fim, como exemplificação prática dos relatos, o modelo de De-Carli et al. ${ }^{5}$ realizou uma pesquisa para analisar o processo de atenção domiciliar nas Equipes de Atenção Básica que aderiram ao Programa Nacional de Melhoria do Acesso e da Qualidade (PNMAQ). A partir de bancos de dados do programa relativos à dimensão que avaliou a realização de visitas domiciliares e cuidados domiciliares, com ênfase nas práticas das Equipes de Saúde Bucal. As visitas domiciliares são realizadas por quase $100 \%$ das Equipes de Saúde da Família. Esse cuidado no domićlio é realizado por quase $90 \%$ dos profissionais da equipe mínima, e por aproximadamente $50 \%$ dos profissionais de Saúde Bucal. Os resultados referentes à realização de visitas domiciliares pelos profissionais da Atenção Básica, tanto Equipe de Saúde da Família quanto nas Unidades Básicas de Saúde no Brasil são de: Médicos 92,5\%; Enfermeiros 99,0\%; Técnico/auxiliar em Enfermagem 94,8\%; Cirurgião-Dentista 56,0\% e Técnico/auxiliar de saúde bucal com 33,6\%. Não houve diferenças significativas entre médicos, enfermeiros e técnicos/auxiliares em enfermagem, porém, comparado com 0 cirurgião-dentista e o técnico/auxiliar de saúde bucal nota-se nitidamente uma grande distância entre as porcentagens. Mostrando que a equipe de saúde bucal tem desafios a serem superados para que possam igualar aos outros profissionais atuantes na ESF, no que se refere em atendimentos domiciliares. Os CDs referem que não realizam a visita domiciliar por não identificarem possibilidades de atuação no espaço do domicílio.

DISCUSSÃO

Percebe-se que com o passar dos anos, foi preciso organizar a base do sistema de saúde, isso porque o Programa Saúde da Família (PSF) gradualmente foi se tornando a principal estratégia para a ampliação do acesso de primeiro contato e de mudança do modelo assistencial. Passando a ser reconhecido como estruturante, isto é, definido como "estratégia de saúde da família". Com isso, em 28 de março de 2006, através da Portaria n.. GM/648, o Ministério da Saúde na gestão do então Ministro José Gomes Temporão, publicou aquilo que é considerado como marco histórico para a consolidação nacional e a expansão da Estratégia de Saúde da Família, visando a reorganização da Atenção Básica no Brasil: a Política Nacional de Atenção Básica (PNAB), alterando e renovando dezenas de Portarias existentes até então. Que foram posteriormente revisada em 2011 e $2017^{9}$.

Diante dos estudos incluídos para síntese, as informações neles contidas são denotam um cenário alarmante, pois contrariam os pressupostos que nortearam a inclusão das Equipes de Saúde Bucal na Atenção Básica, com a proposta de romper com o modelo historicamente baseado no atendimento odontológico clínico individual, excludente e tecnicista ${ }^{7}$. Por exemplo, a integração ensinoserviço-comunidade se mostra crucial para aumentar o número de VD realizadas por CD's à pacientes restritos ao lar no território, mediante ações de prevenção de agravos e promoção da saúde que facilitem a elaboração de um plano de cuidado para cada paciente ${ }^{21}$, indo de encontro com aquilo preconizado pela PNAB para a Equipe de Saúde Bucal, porém diferente daquilo que mais se observa na prática.

Assim, a identificação de um expressivo número de Equipes de Saúde Bucal que não realiza visitas e nem cuidados domiciliares, evidenciado por meio das pesquisas citadas acima, gera inquietação, pois indica que as especificidades do cuidado da Atenção Básica como a equidade de acesso, vínculo, integralidade e a longitudinalidade, não estão sobrevivendo como prática que deveriam ser comum nessas ESF ${ }^{5}$, desse modo contribuindo para a permanência de práticas reducionistas na esfera da ESF, privilegiando as ações curativas e negligenciando o cuidado integral, que deve ser centrado no usuário e principalmente na clínica ampliada ${ }^{5}$.

Isso possivelmente tem acontecido por uma somatória de fatores, como a inserção da Equipe de Saúde Bucal tardia na ESF e à concepção de dependência estrutural de tecnologias duras, associadas ao paradigma biomédico ainda predominante nos processos de formação em odontologia e à dificuldade de trabalhar em equipe e de lidar com ações coletivas $^{21}$. Contudo, é necessário realçar como isso é confrontante com a realidade brasileira, 
de modo que o Brasil tem registrado nas últimas décadas um aumento na expectativa de vida da população, e consequentemente no número de idosos, que são a maior porcentagem daqueles que dependem da atenção no lar. Segundo o IBGE a projeção para o ano de 2040 é de que ocorra uma inversão do número de idosos em comparação ao número de jovens, onde será possível mensurar 153 idosos para cada 100 jovens com idade inferior aos 15 anos. Nessa faixa populacional as principais causas de morte são as DCNT, nas quais se enquadram as patologias cardíacas, cerebrovasculares e as neoplasias ${ }^{16}$.

Com a reorientação do modelo assistencial em 2004, foi lançada a PNAB ampliando o acesso a saúde bucal nos cuidados de todos os níveis de atenção, então as Equipes de Saúde Bucal entraram de vez nas ESF para completar a equipe multiprofissional ${ }^{13}$. Porém, dificilmente o CD consegue sair do consultório da ESF para ir até um domicílio prestar seu serviço, isso ocorre devido à alta demanda de atendimentos clínicos e principalmente demandas espontâneas nas unidades de saúde $^{6}$, além deles não terem uma agenda organizada para ambos os problemas, no que se diz respeito das visitas e dos atendimentos nos consultórios. Outro aspecto que deve ser avaliados são a excessiva demanda reprimidas para a saúde bucal e a incapacidade dos serviços de atenção secundária de a incorporarem a suas agendas, o que acaba por sobrecarregar a Equipe de Saúde Bucal na $\mathrm{ESF}^{5}$.

Outro fator que dificulta essas visitas, citados anteriormente nas pesquisas, é o fato de não terem disponibilidade de equipamentos para atendimentos do tipo home-care ${ }^{22}$. Então quando esses CDs conseguem realizar essas visitas domiciliares, mesmo com as dificuldades apresentadas, os mesmo fazem orientações de saúde bucal e dieta, e salientam o estímulo ao autoexame de câncer de boca ${ }^{18}$. Mais uma condição que relata precariedades a serem superadas pelo CD da ESF, já que a saúde bucal do paciente acamado deve ser avaliada constantemente, pois sua condição oral interfere diretamente em sua qualidade de vida ${ }^{5}$. A falta do acompanhamento do profissional odontólogo pode acarretar em quadros precários de saúde oral que incluem cáries extensas levando ao surgimento de raízes residuais, doenças periodontais e desordens potencialmente malignas ${ }^{4}$.

$\mathrm{O}$ atendimento odontológico domiciliar preventivo e curativo está diretamente relacionado com a melhora do estado de saúde geral do acamado. Ele tem como produto a contribuição para um melhor uso dos leitos hospitalares e da atenção ambulatorial, pois reintegra o paciente em seu núcleo familiar de apoio e favorece a promoção de uma assistência integral e humanizada, por meio do estreitamento do vínculo entre pacienteprofissional $^{20}$.

Quando a visita domiciliar não é vivenciada frequentemente no cotidiano do trabalho na Atenção Básica, o vínculo com a população adscrita fica prejudicado, o que retroalimenta o ciclo do processo de trabalho alienante e isolado dos profissionais da saúde bucal. As visitas domiciliares, sendo inseridas na prática destes profissionais como ações mais frequentes, fomentariam a aproximação com os sujeitos e com a realidade do usuário e da comunidade, bem como otimizariam a força do encontro trabalhador-cuidador-usuário, constituindo-se como uma potência para a transformação das práticas de saúde ${ }^{23}$.

Com isso, as ações inerentes a estas, no âmbito da Atenção Básica, ficam comprometidas, e a atenção integral e a continuidade do cuidado deixam de ser priorizadas perpetuando as práticas de atenção individualizadas no âmbito dos consultórios ${ }^{5}$. Assim, a transformação da realidade requer a transformação dos olhares. A inserção do CD em uma equipe multiprofissional representa um passo nesse sentido, na medida em que os profissionais se somam na construção de novos olhares e o CD amplia suas possibilidades de intervenção, ele reafirma seu papel como profissional da saúde ${ }^{22}$.

CONSIDERAÇÕES FINAIS

A síntese dos dados nos faz compreender melhor a forma pela qual a $A D$ passou a ser atividade inerente ao processo de trabalho das ESF, para possibilitar que pacientes acamados sejam contemplados na integralidade prevista na filosofia do SUS. Também, como a Equipe de Saúde Bucal ainda se mostra numericamente bem abaixo aos demais integrantes da ESF quando a visitas realizadas, e como isso pode ser justificado por complexidades exclusivas a esses - alta demanda de atendimentos clínicos e espontâneos nos consultórios odontológicos da ESF e pela não atuação no espaço do domicílio, por falta de equipamentos adequados do tipo home-care para realização desses atendimentos. Por fim, é necessário que novos modelos sejam estudados de modo a permitir uma presença expressiva de CDs no contexto familiar dos usuários, principalmente dos acamados, favorecendo terão uma assistência 
holística, integral, continuada e humanizada, além de possibilitar a estas pessoas um contato maior com o serviço de saúde. Assim sendo, o impacto na saúde bucal dos acamados devido aos atendimentos domiciliares realizados pelas Equipe de Saúde Bucal nas ESF mostra-se ainda como um importante tema para futuras pesquisas.

\section{REFERÊNCIAS}

1. Reis WG, Scherer MD dos A, Carcereri DL. O trabalho do Cirurgião-Dentista na Atenção Primária à Saúde: entre o prescrito e o real. Saúde em Debate. 2015;39(104):56-64.

2. Brasil. Portaria no 2.436 de 21 de Setembro de 2017. Diário Of da União, Brasília Ministério da Saúde, 21 Set, ed183, Seção 1, p 68; 2017.

3. Rocha DA, Miranda AF. Atendimento odontológico domiciliar aos idosos: uma necessidade na prática multidisciplinar em saúde: revisão de literatura. Rev Bras Geriatr e Gerontol. 2013;16(1):181-9.

4. Cavalcante Maciel JA, Sales de Almeida A, Almeida de Menezes AK, Lima Oliveira Filho I, Macedo Teixeira AK, luco Castro-Silva I, et al. Quando a saúde bucal bate à porta: protocolo para a atenção domiciliar em odontologia. Rev Bras em Promoção da Saúde. 2016;29(4): 614-20.

5. De-Carli AD, Santos ML de M dos, Souza AS de, Kodjaoglanian VL, Batiston AP. Visita domiciliar e cuidado domiciliar na Atenção Básica: um olhar sobre a saúde bucal. Saúde em Debate. 2015;39(105):441-50.

6. Vilarinho SMM, Mendes RF, Prado Júnior RR. Perfil dos cirurgiões-dentistas integrantes do programa saúde da família em Teresina (PI). Rev Odonto Ciência. 2007;22(55):48-54.

7. Mattos GCM, e Ferreira EF, Leite ICG, Greco RM. A inclusão da equipe de saúde bucal na Estratégia Saúde da família: Entraves, avanços e desafios. Cienc saude colet. 2014;19(2): 373-82.

8. Gll AC. Métodos e Técnicas de Pesquisa Social. 6. ed. São. 2008.

9. Pinto LF, Giovanella L. The family health strategy: Expanding access and reducing hospitalizations due to ambulatory care sensitive conditions (ACSC). Cienc saude colet. 2018;23(6):1903913.

10. Savassi LCM. Os atuais desafios da Atenção Domiciliar na Atenção Primária à Saúde: uma análise na perspectiva do Sistema Único de Saúde. Rev Bras Med Família e Comunidade. 2016;11(38):1-12.

11.BRASIL. Protocolo de Atenção Domiciliar em Saúde Bucal. Ministério da Saúde, Sist Único Saúde SUS. 2019;

12. Kátia Bones Rocha, Jaqueline Conz, Mariana Barcinski, Daniel Paiva AP. A visita domiciliar no contexto da saúde: Uma revisão de literatura. Soc Port Psicol da Saúde. 2017;18(1):170-85.

13. Brasil. Departamento de Atenção Básica. Política Nacional de Atenção Básica / Ministério da Saúde. Secretaria de Atenção à Saúde. Departamento de Atenção Básica. Ministério da Saúde; 2012.

14. Brasil. Portaria no 825 de 25 de Abril de 2016. Diário Of da União, Brasília Ministério da Saúde, 25 Abr, ed 78 Seção1, p 33; 2016.

15. Ferreira FPC, Bansi LO, Paschoal SMP. Serviços de atenção ao idoso e estratégias de cuidado domiciliares e institucionais. Rev bras geriatr gerontol. 2014;17(4):911-26.

16. Miranda GMD, Mendes ACG, Cruz A, Silva ALA. O envelhecimento populacional brasileiro: desafios e consequências sociais atuais e futuras. Rev bras geriatr gerontol. 2016; 19(3):507-19.

17. Irineu KN, Acioli Filho JAM, Costa RO, Catão MHCV. Saúde do Idoso e o Papel do Odontólogo: Inter-Relação Entre a Condição Sistêmica e a Saúde Bucal. Rev Faculdade Odontol Lins. 2015;25(2):41-6.

18. Bizerril DO, Saldanha K de GH, Silva JP da, Almeida JR de S, Almeida MEL. Papel do cirurgião-dentista nas visitas domiciliares: atenção em saúde bucal. Rev Bras Med Família e Comunidade. 2015;10(37):1-8.

19. Brasil. Diretrizes da Política Nacional de Saúde Bucal. Ministério da Saúde Secr Atenção à Saúde Dep Atenção Básica Coord Nac Saúde Bucal. 2004;

20. Costa Marques Fernando Luiz Brunetti Montenegro Luciana Freitas Bezerra Eric Jacomino Franco Aline Úrsula Rocha Fernandes Alexandre Franco Miranda G. Odontologia domiciliar ao idoso frágil: a importância da Odontogeriatria "Como Publicado na. Rev Portal Divulg. 2014;42(5): 6-14.

21. Pereira de Lima A, Sousa Lopes T, Aguiar de Lima AF, Ramalho de Farias MR de F, Cavalcante Maciel JA. Atenção Domiciliar Em Saúde Bucal: Experiência De Integração Ensino-Serviço-Comunidade Em Centro De Saúde Da Família. SANARE - Rev Políticas Públicas. 2019;18(1):90-7.

22. Sebold R, Carcereri DL. Processo de trabalho em saúde bucal: em busca de diferentes olhares para compreender e transformar a realidade Work process in oral health: seeking different looks to understand and transform the reality. Cien saude colet. 2010;15:1643-52.

23. David HMSL, Acioli S, Silva MRF, Bonetti OP, Passos H. Pandemia, conjunturas de crise e prática profissional: qual 0 papel da enfermagem diante da Covid-19? Rev Gaúcha Enferm.2021;429(Spe):1-7. 


\section{CONFLITO DE INTERESSES}

Os autores declaram não haver conflitos de interesse

\section{AUTOR PARA CORRESPONDÊNCIA}

\section{Sara de Oliveira Rosa}

Faculdade de Odontologia,

Universidade Federal de Alfenas (UNIFAL-MG)

Rua Gabriel Monteiro da Silva, 700

37130-001 Alfenas-MG, Brasil

E-mail: saah.oliveira@hotmail.com

\section{Submetido em 04/04/2021}

Aceito em 16/07/2021 